by $B$ cells through affecting monocytes. IL- 6 and/or IL-10 may intermediate the effect. Our findings strongly suggest that BAFF signaling via BR3 is a possible therapeutic target for drug discovery to treat pSS or other intractable autoimmune diseases which accompany hypergammaglobulinemia. Moreover, these compounds may provide novel tools to explore the pathological mechanism of the development of these autoimmune diseases.

Disclosure of Interest: K. Yoshimoto: None declared, K. Suzuki: None declared, K. Sugahara Employee of: Mitsubishi Tanabe Pharma Corporation, T. Takeuchi Grant/research support from: Mitsubishi Tanabe Pharma Corporation

DOI: 10.1136/annrheumdis-2017-eular.4176

\section{THU0056 FREE FATTY ACIDS PROMOTE INFLAMMATION VIA OSTEOBLASTS AND OSTEOCLASTS FROM PATIENTS WITH RHEUMATOID ARTHRITIS OR OSTEOARTHRITIS}

K. Frommer ${ }^{1}$, A. Schäffler ${ }^{2}$, U. Lange ${ }^{1}$, S. Rehart ${ }^{3}$, J. Steinmeyer ${ }^{4}$ M. Rickert ${ }^{4}$, U. Müller-Ladner ${ }^{1}$, E. Neumann ${ }^{1} \cdot{ }^{1}$ Dept. of Internal Medicine and Rheumatology, Kerckhoff-Klinik, Justus-Liebig-University of Giessen, Bad Nauheim; ${ }^{2}$ Dept. of Internal Medicine III, Endocrinology, Diabetes, Metabolism, Justus-Liebig-University of Giessen, Giessen; ${ }^{3}$ Dept. of Orthopaedics and Trauma Surgery, Agaplesion Markus Hospital, Frankfurt; ${ }^{4}$ Dept. of Orthopaedics and Orthopaedic Surgery, University Hospital Giessen and Marburg, Giessen, Germany

Background: Various inflammatory cardiovascular and metabolic diseases such as atherosclerosis, coronary heart diseases and type 2 diabetes are associated with chronically elevated free fatty acid (FFA) levels. With inflammation being a factor in pathological bone loss, FFA may also be contributors to bone loss in osteoarthritis (OA) and/or rheumatoid arthritis (RA).

Objectives: To investigate whether FFA have an influence on osteoblasts and osteoclasts from patients with RA or OA, in a way that may alter bone degradation in these diseases.

Methods: Primary osteoblasts $(\mathrm{OB})$ were isolated from cancellous bone of $\mathrm{OA}$ and RA patients undergoing knee joint surgery. Osteoclasts $(\mathrm{OC})$ were differentiated from peripheral blood mononuclear cells (PBMC). $\mathrm{OB}$ and $\mathrm{OC}$ were stimulated with the saturated FFA palmitic acid (PA) and the unsaturated FFA linoleic acid (LA) (100 $\mu \mathrm{M}$ each). Immunoassays were used to quantify protein secretion. mRNA expression levels were quantified by real-time PCR. Mineralization activity was quantified using Alizarin Red S staining, differentiated OC were quantified by counting TRAP-positive multinuclear cells (>2 nuclei). Toll-like receptor (TLR) 4 and TLR2 were blocked by neutralizing antibodies.

Results: When stimulated with FFA, OB from RA and OA patients secreted higher amounts of the proinflammatory cytokine IL-6 (up to 9-fold) and the chemokines IL-8 (up to 221-fold), GRO-a (from below detection level to detectable levels) and MCP-1 (up to 16-fold). Differences in the degree of response were more dependent on the patient than the disease. RANKL as well as OPG, OB-secreted modulators of OC differentiation, as well as $\mathrm{OB}$ differentiation markers (e.g. osterix, osteocalcin) were not influenced by FFA on mRNA or protein level. The effect of FFA on mineralization activity of $\mathrm{OB}$ varied between patients, yet overall there was no significant difference between FFA-treated and untreated $\mathrm{OB}$. Expression of the two Wnt signaling molecules, axin-2 and b-catenin, was not changed by PA or LA, suggesting no involvement of the Wnt signaling pathway in the effects observed by FFA in OB. On the other hand, TLR4 blockade significantly reduced PA-induced IL-8 secretion by OB (by 93\%), while blocking TLR2 had no effect. In both RA and OA OC, IL-8 secretion was significantly enhanced by PA and LA, with a clear time-dependency within the differentiation process for RA OC but not for OA OC. The number of TRAP positive multinuclear cells decreased for RA OC by approx. $50 \%$, which was in agreement with the reduced TRAP secretion by a factor of $2-3$ at d14. mRNA expression of various osteoclast activity markers (CLCN7, CTSK, TCIRG) was not altered.

Conclusions: Inflammation is promoted by FFA via both $\mathrm{OB}$ and $\mathrm{OC}$ from patients with RA or OA, thus possibly indirectly contributing to bone loss while no direct effect on $\mathrm{OB} / \mathrm{OC}$ activity could be observed. In $\mathrm{OB}$, these effects are probably mainly mediated by TLR4, while TLR2 and Wnt pathways do not play a role.

Disclosure of Interest: None declared

DOI: 10.1136/annrheumdis-2017-eular.3488

\section{THU0057 LIPID PROFILING OF PLASMA IN RHEUMATOID ARTHRITIS PATIENTS BY LIQUID CHROMATOGRAPHY-TANDEM MASS SPECTROMETRY}

K. Murakami ${ }^{1}$, I. Murakami ${ }^{1}$, A. loan-Facsinay ${ }^{2}$, M. Giera ${ }^{3}$, M. Hashimoto ${ }^{4}$ A. Yoshida ${ }^{1}$, T. Usui ${ }^{1}$, N. Kuramoto ${ }^{1}$, R. Nakashima ${ }^{1}$, Y. Imura ${ }^{1}$, H. Yoshifuji ${ }^{1}$, K. Ohmura ${ }^{1}$, T. Mimori ${ }^{1} .{ }^{1}$ Department of Rheumatology and Clinical Immunology, Graduate School of Medicine, Kyoto University, Kyoto, Japan; ${ }^{2}$ Department of Rheumatology; ${ }^{3}$ Center for Proteomics and Metabolomics, Leiden University Medical Center, Leiden, Netherlands; ${ }^{4}$ Department of the Control for Rheumatic Diseases, Graduate School of Medicine, Kyoto University, Kyoto, Japan

Background: Previously it has been described that lipid and lipid mediators are present in synovial fluid from patients with rheumatoid arthritis (RA). It is, however, currently unknown to what extent these lipid mediators are involved in disease pathophysiology.

Objectives: The aim of this study is to clarify which lipid mediators in plasma correlate with disease activity of RA.

Methods: We obtained blood from RA patients registered in the KURAMA (Kyoto University Rheumatoid Arthritis Management Alliance) cohort. None of the patients was treated with glucocorticoids or NSAIDs, both of which could affect lipid metabolism. Targeted lipidomics, using a LC-MS/MS (liquid chromatographytandem mass spectrometry) platform was used for the identification of lipids present in the patients' plasma. SDAI (simplified disease activity index) was examined in this cohort and lipidomics profiling and disease status were combined. Data were statistically analyzed by Spearman's rank correlation coefficient test or multivariate regression analysis.

Results: Twenty-six RA patients were enrolled; female ratio: $84 \%$, mean age: 63.0 years old, mean disease duration: 18.7 years and mean SDAI 5.26. In this group, patients age was significantly correlated with SDAI ( $p$ value $=0.005$, Spearman's rho $=0.552$ ). By LC-MS/MS analyses, 23 lipid components were identified and quantified. Multivariative regression analysis (Standard Least Squares) revealed that 19,20-diHDPA (Dihydroxydocosapentaenoic acid) and 14,15-diHETE (Dihydroxyeicosatetraenoic acid) significantly explained SDAI score independently of sex and age.

Among the composite measure for SDAI, the best correlated component with TJC (tender joint count) was LA (Linoleic acid, $p=0.002$, rho $=-0.611$ ), that with patient VAS (visual analogue scale) was 19,20 -diHDPA $(\mathrm{p}=0.032$, rho $=0.440)$, and that with CRP was DHA (Docosahexaenoic acid, $\mathrm{p}=0.021$, rho $=-0.452$ ).

Additionally, principal component analysis was carried out. In the first primary component (PC1), absolute eigenvecotor values of AdA (Adrenic acid), ALA (Alpha-linolenic acid), DHA, DPA (Docosapentaenoic acid) and LA are more than 0.25 , among which DHA was strongly correlated with PC1 $(p<0.0001$, rho $=0.902$ ). PC1 positively and significantly explained TJC count independent of sex and age.

\begin{tabular}{llllll}
\multicolumn{5}{c}{ Table. Parameter } & Estimates by Standard Least Squares \\
& Lower $95 \%$ & Upper $95 \%$ & Std. Beta & p value \\
\hline Intercept & -39.2 & -6.63 & 0 & 0.008 \\
19,20-diHDPA & 4.54 & 16.55 & 0.707 & 0.003 \\
14,15-diHDPE & -26.4 & -6.24 & -0.62 & 0.003 \\
Age & 0.08 & 0.56 & 0.455 & 0.013 \\
Sex & & & & 0.475
\end{tabular}

Conclusions: Since 19, 20-diHDPA (metabolized from DHA) and 14,15-diHETE (from EPA, eicosapentaenoic acid) are both generated by cytochrome P450catalyzed epoxidation followed by conversion to the vicinal diols by epoxide hydrolase, such kind of enzymes might be key molecules connecting lipid metabolism and RA. Although a replication study is inevitable, a certain kinds of lipid and lipid mediator profiles may be associated with disease activity, especially analgesic descriptors such as tender joint count.

References:

[1] Giera M, et al. Lipid and lipid mediator profiling of human synovial fluid in rheumatoid arthritis patients by means of LC-MS/MS. Biochim Biophys Acta. 2012, 1821(11):1415-24.

Acknowledgements: None.

Disclosure of Interest: None declared

DOI: 10.1136/annrheumdis-2017-eular.4052

\section{THU0058 S100A11 PROTEIN IS UP-REGULATED IN PATIENTS WITH IDIOPATIC INFLAMMATORY MYOPATHIES AND IS ASSOCIATED WITH DISEASE ACTIVITY}

L. Andres Cerezo ${ }^{1}$, H. Hulejová ${ }^{1}$, B. Šumová ${ }^{1}$, T. Lennerovaa ${ }^{1}$, M. Klein ${ }^{1}$, H.F. Mann ${ }^{1}$, J. Zámečník ${ }^{2}$, K. Pavelka ${ }^{1}$, J. Vencovský ${ }^{1}$, L. Šenolt ${ }^{1}$. ${ }^{1}$ Department of Rheumatology, 1st Faculty of Medicine, Charles University Prague, Institute of Rheumatology; ${ }^{2}$ Department of Pathology and Molecular Medicine, 2nd Faculty of Medicine, Charles University Prague, University Hospital Motol, Prague, Czech Republic

Background: S100A11 (calgizzarin) is a member of the S100 protein family that participates in regulating number of biologic functions and is associated with oncogenesis and inflammation. Recent data suggest involvement of S100A11 in myocardial damage.

Objectives: The aim of our study was to analyze the expression of S100A11 in patients with idiopathic inflammatory myopathies (IIMs) and its potential association with disease activity parameters and IIMs-related clinical features.

Methods: immunohistochemistry in patients with polymyositis/dermatomyositis (PM/DM, $n=5 / 6)$ and control individuals with myasthenia gravis (MG, $n=5$ ). S100A11 in plasma was measured by ELISA (Biovendor) in 112 patients with IIMs (PM, $n=41 ; D M, n=41$; and cancer associated myositis (CAM), $n=30$ ) and in 42 healthy controls $(\mathrm{HC})$. Patients with PM/DM fulfilled Bohan and Peter diagnostic criteria and CAM was defined as cancer occurring within 3 years of the diagnosis of myositis. Clinical disease activity was assessed by myositis disease activity assessment (MYOACT), physician and patient's global activity using visual analogue scales (VAS), manual muscle testing (MMT) and health assessment questionnaire (HAQ). Muscle enzymes CK, LD, ALT, AST and CRP were measured by routine laboratory techniques. Autoantibodies were detected by immunoprecipitation. 
Results: S100A11 protein was up-regulated in the muscle from patients with IIMs compared to MG. In PM/DM patients, S100A11 was accumulated in the cytoplasm of regenerating and necrotizing muscle fibers and on the sarcolemma of most fibers. Only some mononuclear infiltrate cells showed S100A11 positivity. In patients with MG, S100A11 was detected on the sarcolemma only. Moreover, S100A11 was increased in plasma of patients with IIMs compared to HC (3.82 [1.47-72.9] vs. $2.84[1.67-11.18] \mathrm{ng} / \mathrm{ml} ; \mathrm{p}=0.0076)$. When divided into groups, patients with DM and CAM showed significant elevation of plasma S100A11 compared to HC (4.10 [2.22-50.81] and 4.52 [1.53-9.11] vs. 2.84 [1.67-11.18] $\mathrm{ng} / \mathrm{ml} ; \mathrm{p}=0.004$ and $\mathrm{p}=0.02$, respectively). Levels of $\mathrm{S} 100 \mathrm{~A} 11$ did not differ between PM and HC. In all patients, S100A11 correlated with LD ( $r=0.300$, $p=0.001)$, CK $(r=0.217, p=0.025)$ and AST $(r=0.343, p<0.001)$, with MYOACT $(r=0.279, p=0.005)$ and with muscle, pulmonary and skin disease activity $(r=0.199$, $p=0.040 ; r=0.224, p=0.025$ and $r=0.307, p=0.001)$. S100A11 levels were elevated in autoantibody-positive group compared to autoantibody-negative group of patients with IIMs (5.22 [1.45-69.29] vs 3.44 [1.58-12.12], $\mathrm{p}=0.010)$.

Conclusions: S100A11 is up-regulated in the circulation of patients with IIMs and correlates with disease activity. Accumulation of S100A11 in the muscle of patients with myositis may indicate its potential role in the process of cell necrosis and fiber regeneration.

Acknowledgements: Supported by grant 15-34065A of the Agency for Healthcare Research of the Czech Republic and MHCR 023728.

Disclosure of Interest: None declared

DOI: 10.1136/annrheumdis-2017-eular.4758

\section{THU0059 ONCOSTATIN M INDUCES INFLAMMATION AND DIFFERENTIALLY REGULATES TNF ALPHA-INDUCED PRO-INFLAMMATORY MECHANISMS AND NOTCH SIGNALLING IN THE RA JOINT}

M.M. Hanlon ${ }^{1}$, D. Veale ${ }^{2}$, S. Wade ${ }^{1}$, M. Biniecka ${ }^{2}$, U. Fearon ${ }^{1}$, T. McGarry ${ }^{1}$ ${ }^{1}$ Molecular Rheumatology, Trinity Biomedical Sciences Institute, Trinity College Dublin; ${ }^{2}$ Centre for Arthritis and Rheumatic Diseases, St Vincents University Hospital, University College Dublin, Dublin, Ireland

Background: Oncostatin M (OSM) is a pleiotropic cytokine, highly expressed in the RA joint that displays both agonistic and antagonistic effects depending on the inflammatory microenvironment. This study examines the effect of OSM on inflammation, the Notch-1 signalling pathway which plays a critical role in vascular development and angiogenesis and finally on TNF $\alpha$-induced pro-inflammatory mechanisms in Rheumatoid Arthritis.

Objectives: To examine the effect of OSM on cytokine/chemokine production, angiogenesis and the Notch-1 signalling pathway in synovial fibroblasts and endothelial cells and whether OSM potentiates the effects of TNF $\alpha$-induced pro-inflammatory effects.

Methods: Primary RA synovial fibroblasts (RASFC) isolated from RA synovial biopsies obtained at time of knee arthroscopy and human dermal microvascular endothelial cells (HMVEC) were grown to confluence. RASFC and HMVEC were cultured with OSM (10ngml) alone or in combination with increasing concentrations of TNF $\alpha(0.01-1 \mathrm{ng} / \mathrm{ml})$. IL-6, IL-8, RANTES, GRO $\alpha$ and MCP1 cytokines/chemokines were quantified in culture supernatants by ELISA. Functionally, angiogenesis and invasion were assessed by matrigel tube formation and Transwell invasions assays respectively, and VEGF in cell lysates quantified by Real-time PCR. Finally, Notch-1, its ligands Delta-like-ligand 4 (DLL-4) and Jagged-1 (Jag-1) and downstream transcriptional repressors - Hey-1 and Hey-2 were quantified by Real-time PCR.

Results: OSM alone significantly induced IL-6 and MCP-1 while inhibiting IL-8 and GRO $\alpha$ in RASFC and HMVEC culture supernatants, compared to basal control. OSM alone induced RANTES expression in HMVEC with little effect observed for RASFC. OSM potentiated the effect of increasing concentrations of TNF $\alpha$ on IL- 6 and MCP-1 secretion from RASFC and HMVEC. Conversely OSM significantly inhibited TNF $\alpha$-induced IL-8 and GRO $\alpha$ secretion from both RASFC and HMVEC. Interestingly, OSM significantly inhibited TNFa-induced RANTES expression in HMVEC yet conversely potentiated this effect in RASFC. At a functional level, OSM induced both RASFC and HMVEC invasion and induced network formation and VEGF expression in HMVEC. OSM significantly induced Notch-1 in RASFC and HMVEC in a time dependent manner, but interestingly differentially regulated the Notch-1 ligands, with induction of Jag-1 only observed in RASFC, and induction of DLL-4 only observed in HMVEC. Finally OSM differentially regulated Notch-1 downstream transcriptional repressors in HMVEC, significantly inducing Hey-2 while simultaneously inhibiting Hey-1.

Conclusions: OSM is a pleiotropic cytokine that displays divergent effects with both pro- and anti-inflammatory mechanisms within the inflamed joint, effects that appear to be dependent on cell type and the inflammatory microenvironment. Targeting OSM or downstream signalling pathways may lead to new potential therapeutic strategies or adjuvant therapies, particularly for those patients who have sub-optimal responses.

Disclosure of Interest: None declared

DOI: 10.1136/annrheumdis-2017-eular.6334

\section{THU0060 T CELL-DERIVED IL-17A AND IL-17F DRIVE BONE FORMATION FROM HUMAN PERIOSTEAL STEM CELLS: IMPLICATIONS FOR ENTHESOPHYTE FORMATION}

M. Shah ${ }^{1}, 2$, A. Maroof ${ }^{1}$, R. Al-Hosni ${ }^{3}$, P. Gikas ${ }^{4}$, N. Gozzard ${ }^{1}$, S. Shaw ${ }^{1}$ S. Roberts ${ }^{1,2}$. ${ }^{1}$ UCB Pharma, Slough; ${ }^{2}$ University College London; ${ }^{3}$ Institute of Orthopaedics and Musculoskeletal Science; ${ }^{4}$ The Royal Orthopaedic Hospital, London, United Kingdom

Background: Pathological bone formation associated with spondyloarthropathies $(\mathrm{SpA})$ is a major cause of structural tissue damage causing permanent disability. A paucity of in vitro models that faithfully replicate human skeletal biology has impeded research into the cellular and molecular triggers for this osteoimmunological phenomenon. Nevertheless, clinical and animal studies have defined IL-17 signalling as a key regulator of $\mathrm{SpA}$ disease; however, the role of IL-17 in bone pathology is unclear. IL-17-producing $\gamma \delta$-T cells have a critical function in periosteal bone formation for fracture repair ${ }^{1}$. The periosteum has also been implicated in pathological bone formation during SpA disease progression².

Objectives: To investigate IL-17 signalling in the context of pathological bone formation using a biomimetic human periosteum derived stem cell (hPDSC) model of osteogenic differentiation.

Methods: hPDSCs were obtained through enzymatic digestion of periosteal biopsies from patients undergoing orthopaedic surgery. Expanded cultures were then treated with recombinant human IL-17A, IL-17F, or both over 96h. The expression of gene markers was then evaluated. Alternatively, hPDSCs were stimulated using a biomimetic protocol in combination with IL-17A and IL-17F, or human T-cell supernatants (SNs) (as a surrogate disease-like inflammatory milieu). Antibodies with strong-affinity to IL-17A, IL-17F, or bimekizumab (a humanised monoclonal IgG1 antibody with strong affinity for both IL-17A and IL-17F) were used to define the role of these cytokines in the SNs. Expression of osteogenic markers and matrix mineralisation was assessed to define in vitro bone formation.

Results: Under basal conditions IL-17A and IL-17F significantly up-regulated IL-6 expression. Additionally, IL-17A and IL-17F transiently enhanced the expression of the osteogenic transcription factor RUNX-2. When IL-17 cytokines were combined in a biomimetic differentiation protocol, both IL-17A and IL-17F promoted osteogenic differentiation. Importantly, IL-17F enhanced the expression of most osteogenic markers to a greater extent than IL-17A alone following 9 days' exposure. Conversely, IL-17A treatment resulted in elevated in vitro mineralisation vs IL-17F. Th17 and $\gamma \delta-T$ cell SNs potently enhanced hPDSC osteogenic differentiation and mineralisation. Whilst $I L-6$ expression and in vitro bone formation were blocked by neutralisation of IL-17A or IL-17F, dual neutralisation of IL-17A and IL-17F in the inflammatory milieu exhibited the greatest effect on most of the tested parameters. Conclusions: These data show that both IL-17A and IL-17F enhance in vitro osteogenic differentiation and bone formation from hPDSCs. The source of these cytokines has not been established but is likely to involve entheseal resident $\gamma \delta$-T cells. We propose that following their release, IL-17A and IL-17F drive pathological bone formation resulting in enthesophytes at the enthesis/periosteum interface. Current therapeutics display limited efficacy in blocking enthesophyte formation, hence inhibition of both IL-17A and IL-17F offers an attractive therapeutic strategy to prevent this debilitating feature of $\mathrm{SpA}$.

References:

[1] Ono, et al. Nat Commun 2016;7:10928.

[2] Lories, et al. Arthritis Res Ther 2009;11:221.

Disclosure of Interest: M. Shah Grant/research support from: UCB Pharma, Employee of: UCB Pharma, A. Maroof Employee of: UCB Pharma, R. Al-Hosni: None declared, P. Gikas: None declared, N. Gozzard Employee of: UCB Pharma, S. Shaw Employee of: UCB Pharma, S. Roberts Employee of: UCB Pharma DOI: 10.1136/annrheumdis-2017-eular.3826

\section{THU0061 REGULATION OF OSTEOCLAST RECRUITMENT AND ACTIVATION BY RESOLVIN D1}

H.A. Benabdoune, P. Rondon, Q. Shi, J.C. Fernandes, H. Fahmi, M. Benderdour Research Centre, Sacré-Coeur Hospital of Montreal, Room K-3045, Montreal, Canada

Background: Resolvin-D1 (RvD1) is a derivative of omega-3 fatty acids and a potent anti-inflammatory agent synthesized during the resolution phase of inflammation. In human cartilage, we recently reported that RvD1 strongly inhibited a number of factors involved in inflammation, catabolism, oxidative stress, and apoptosis.

Objectives: The overall objective of this study is to further investigate RvD1 effects on bone metabolism in vitro.

Methods: First, murine macrophages RAW267.4 were used to assess osteoclasts (OC) recruitment and bone resorption. RAW264.7 cells were incubated with 50 $\mathrm{ng} / \mathrm{ml}$ LPS with or without RvD1 $(0-10 \mu \mathrm{M})$ for 48 hours. Cell viability was verified with the MTS test. OC phenotype markers, namely TRAP and cathepsin-K, were assessed by western blot, enzymatic staining and immunocytochemistry. Levels of TNF- $\alpha$, IL-1 $1 \beta$, IL-6, IL-10, were measured by ELISA, and PGE 2 levels by EIA. NO release was measured by Greiss reaction.

To investigate bone resorption, RAW264.7 cells were seeded in hydroxyapatite plates, then treated with $50 \mathrm{ng} / \mathrm{ml}$ LPS with or without RvD1 $(0.5$ and $1 \mu \mathrm{M})$ for 48 hours. Plot formation was assessed by Von Kossa staining. 